\title{
Design of a Power-Efficient Widely-Programmable Gm-LC Band-Pass Sigma-Delta Modulator for SDR
}

\author{
Alonso Morgado, Rocío del Río and José M. de la Rosa \\ Instituto de Microelectrónica de Sevilla, IMSE-CNM (CSIC/Universidad de Sevilla) \\ C/Américo Vespucio, 41092 Sevilla, SPAIN \\ E-mail: [alonso,rocio,jrosa]@imse-cnm.csic.es
}

\begin{abstract}
This paper presents the design and implementation of a fourth-order band-pass continuous-time $\Sigma \Delta$ modulator intended for the digitization of radio-frequency signals in softwaredefined-radio applications. The modulator architecture consists of two Gm-LC resonators with a tunable notch frequency and a 4-bit flash analog-to-digital converter in the forward path and a non-return-to-zero digital-to-analog converter with a finiteimpulse-response filter in the feedback path. Both system-level and circuit-level reconfiguration techniques are included in order to allow the modulator to digitize signals placed at different carrier frequencies, from $450 \mathrm{MHz}$ to $950 \mathrm{MHz}$. A proper synthesis methodology of the loop-filter coefficients at system level and the use of inverter-based switchable transconductors allow to optimize the performance in terms of robustness to circuit errors, stability and power consumption. The circuit, implemented in 65nm CMOS, can digitise signals with up to 57-dB SNDR within a 40-MHz bandwidth, with an adaptive power dissipation of 16.7to-22.8 $\mathrm{mW}$ and a programmable $1.2 / 2 \mathrm{GHz}$ clock rate ${ }^{1}$.
\end{abstract}

\section{INTRODUCTION}

Continuous-Time $\Sigma \Delta$ Modulators (CT- $\Sigma \Delta \mathrm{Ms}$ ) are proving to be a competitive solution for the implementation of powerefficient Analog-to-Digital Converters (ADCs) operating in the $\mathrm{GHz}$ range, thus opening the door to use them in digitalintensive Radio-Frequency (RF) transceivers and Software Defined Radio (SDR) [1]-[7]. One of the trends to implement such SDR receivers consists of placing a Band-Pass (BP) $\mathrm{CT}-\Sigma \Delta \mathrm{M}$ as close as possible to the antenna, yielding to the so-called $R F$-to-digital converters [1], [4], [6], [8]-[10]. These $\Sigma \Delta \mathrm{M}$-based RF digitizers include diverse strategies to reduce their aggressive specifications in terms of the sampling frequency $\left(f_{s}\right)$ and the Dynamic Range (DR), including, among others, embedded out-of-band filtering [1], frequencytranslating and undersampling or subsampling [6]. The latter allows to digitize RF signals placed at $f_{\mathrm{RF}}>f_{s} / 2$, while keeping high values of the OverSampling Ratio (OSR), since the signal bandwidth is typically $B_{w} \ll\left(f_{\mathrm{RF}}, f_{s}\right)$. Other authors have proposed the use of a tunable center frequency or notch frequency in order to simplify the design of the frequency synthesizer needed in the RF receiver [4], [6], [11].

In spite of their potential benefits, the performance of CT$\Sigma \Delta$ RF-to-digital converters is still well short of what is

\footnotetext{
${ }^{1}$ This work was partially supported by the Spanish Ministry of Economy and Competitiveness (with support from the European Regional Development Fund) under contracts TEC2010-14825/MIC and TEC2013-45638-C3-3-R, and by "Consejería de Economía, Innovación, Ciencia y Empleo de la Junta de Andalucía", under contract P12-TIC-1481.
}

needed for comercialization. The main challenge is associated with the design of the loop filter, so that a high-quality and accurate resonance can be achieved, while keeping the necessary tuning range, robustness and stability. Thus, LC tanks are great from a power and linearity perspective but typically support a reduced range, whereas active-RC resonators can be widely tunable but require amplifiers with high gain at the ADC center (RF carrier) frequency.

One of the key points to address these challenges is to optimize the design of BP CT- $\Sigma \Delta \mathrm{Ms}$ by properly selecting the modulator loop-filter coefficients in terms of tunnability, resonator input/output swing and simplicity of the circuit implementation. This paper contributes to this topic and describes the design and layout-level implementation of a fourthorder Gm-LC BP CT- $\Sigma \Delta \mathrm{M}$ in a $65-\mathrm{nm}$ CMOS technology. A systematic methodology is used to optimize the loopfilter design in order to reduce the power consumption to be competitive with conventional baseband digitization, while keeping the benefits of RF digitization. Simulation data feature 9-bit effective resolution for $40-\mathrm{MHz}$ signals placed at $450-$ to-950 $\mathrm{MHz}$ with scalable 16.6 -to- $22.7 \mathrm{~mW}$ power dissipation.

\section{Modulator Architecture}

Fig. 1 shows the modulator architecture. It consists of a fourth-order BP CT- $\Sigma \Delta \mathrm{M}$ with multi-bit ( $B=4$ bit) quantizer and a loop filter made up of two LC-based resonators with a transfer function, $R(s)=\omega s /\left(s^{2}+\omega^{2}\right)$, where normalized values of $s$ and $\omega$ are considered with respect to $f_{s}$, so that $s=2 \pi f / f_{s}$ and $\omega \equiv 2 \pi f_{n} / f_{s}$. For synthesis purposes, the forward-path coefficients are defined as $k_{1,2}=k / S f R_{1,2}$ and $k_{3}=1 /\left(S f R_{1} \cdot S f R_{2}\right)$, where $k$ is a coefficient used to equalize the Signal Transfer Function (STF) and $S f R_{1,2}$ are the scaling caling coefficients which modify the resonator gain, $R_{\text {gain }}=\omega \cdot s[11]$. The feedback path consists of a NonReturn-to-Zero (NRZ) Digital-to-Analog Converter (DAC) and a two-tap half-delayed Finite-Impulse-Response (FIR) filter, with scaling coefficients, $c_{i}$ and $c_{i d}$, that provide the necessary degrees of freedom in the synthesis of the Noise Transfer Function (NTF) when applying a CT-to-DT equivalence. Additional feedback paths with scaling gains, $c_{0 j}$, are included to compensate for the Excess Loop Delay (ELD) error. 


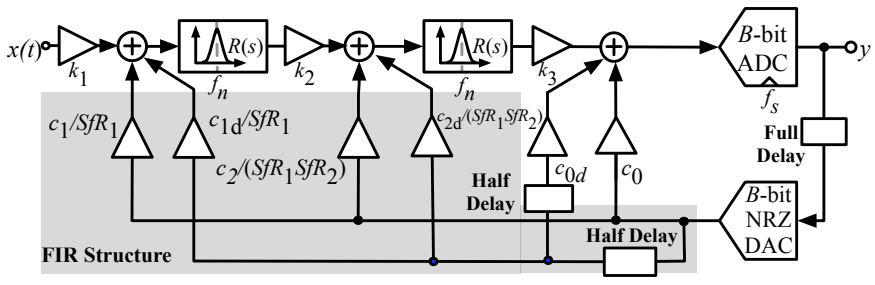

Fig. 1. Block diagram of the fourth-order BP CT- $\Sigma \Delta \mathrm{M}$.

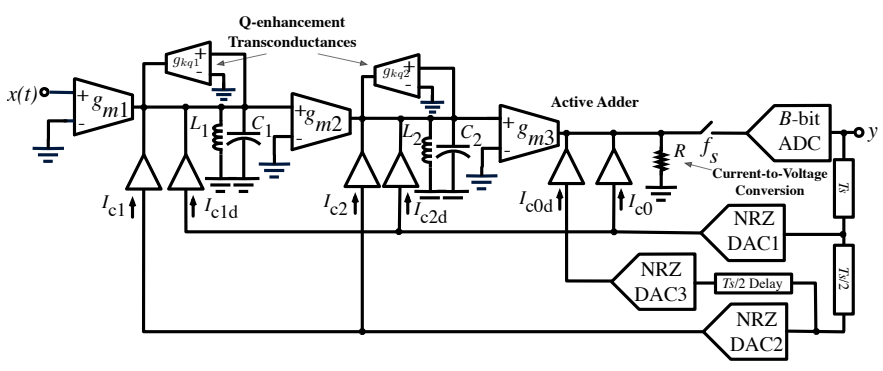

Fig. 2. Conceptual Gm-LC schematic of the proposed modulator.

\section{A. Gm-LC Implementation}

The BP CT- $\Sigma \Delta \mathrm{M}$ in Fig. 1 can be implemented using any arbitrary CT circuit technique, either active-RC or Gm-LC. In this work, a Gm-LC implementation - conceptually depicted in Fig. 2 - has been considered, where $g_{m 1,2}=k_{1,2} \cdot C \cdot \omega \cdot f_{s}$, $g_{m 3}=k_{3} / R, I_{c 0, c 0 d}=\left(c_{0}, c_{0 d}\right) \cdot V_{\mathrm{FS}}, I_{c 1, c 1 d}=\left(c_{1}, c_{1 d}\right)$. $g_{m 1} \cdot V_{\mathrm{FS}}, I_{c 2, c 2 d}=\left(c_{2}, c_{2 d}\right) \cdot g_{m 2} \cdot V_{\mathrm{FS}} / S f R_{1}$, and $V_{\mathrm{FS}}=1 \mathrm{~V}$ stands for the full-scale reference voltage. A fully differential version of this circuit - not shown in Fig. 2 for simplicity is implemented in practice. All transconductors are realized as multiples of a unitary transconductance element, $g_{m u}$. The quality factor, $Q$, of $\mathrm{Gm}$ - $\mathrm{LC}$ resonators is enhanced by adding two extra transconductances, $g_{k q 1,2}$. The 4-bit quantizer is made up of a flash ADC in the loop-filter forward path and a NRZ current-steering FIR-DAC in the feedback path.

\section{B. High-Level Synthesis}

The modulator in Fig. 2 has been synthesized by applying a CT-to-DT transformation to a BP DT- $\Sigma \Delta \mathrm{M}$ with a NTF which satisfies the required specifications in terms of DR and signal bandwidth, $B_{w}$. To this end, the Schreier's toolbox is used to synthesize the NTF for a given value of $f_{n}$ and Out-of-Band Gain (OBG), and the modulator loop-filter is derived from the impulse-invariant transformation. Once the ideal NTF and STF have been synthesized, the best values of the loop-filter coefficients are selected in order to optimize the performance of the BP CT- $\Sigma \Delta \mathrm{M}$ in terms of robustness and stability, while maximizing DR and the Signal-to-(Noise+Distortion) Ratio (SNDR) with the minimum power consumption, $P$.

The values of DR and SNDR can be computed from simulation by using SIMSIDES [12] and $P$ can be approximately estimated as the sum of the power consumed by resonators, $P_{\text {RES }}$, by the active adder, $P_{\mathrm{ADD}}$, and by the embedded quantizer, i.e. the ADC and the DAC. The latter is estimated from electrical simulations from Cadence Spectre ${ }^{\circledR}$, whereas $P_{\mathrm{RES}}$ and $P_{\mathrm{ADD}}$ are respectively derived as:

$$
\begin{aligned}
& P_{\mathrm{RES}}=V_{\mathrm{DD}} \cdot\left[\sum_{i=1}^{2}\left(I_{c i}+I_{c i d}\right)+\frac{g_{m 1}+g_{m 2}}{g_{m I D}}\right] \\
& P_{\mathrm{ADD}}=V_{\mathrm{DD}} \cdot\left[\frac{g_{m 3}}{g_{m I D}}+\frac{c_{0}+c_{0 d}}{R} \cdot V_{\mathrm{FS}}\right]
\end{aligned}
$$

where $V_{\mathrm{DD}}$ is the supply voltage and $g_{m I D}$ stands for the transconductance-versus-current efficiency. In this design, the same capacitances, $C_{1}=C_{2}=C$, inductances, $L_{1}=L_{2}=L$, and $Q$-enhancement gains, $g_{k q 1}=g_{k q 2}=g_{k q}$, are assumed for both resonators.

Given a set of specifications defined in terms of SNDR, $B_{w}$ and $P$, the optimization procedure can be formulated as a design problem with five design variables, namely: $\mathrm{OBG}, R_{\text {gain }}$, $S f R_{1,2}$ and $g_{k q}$. In order to optimize these design variables, the following step-by-step procedure has been followed:

- Step 1, in which parameters $R_{\text {gain }}$ and OBG are optimized based on a parametric simulation analysis, where $R_{\text {gain }}$ and OBG are swept, and the rest of parameters are fixed to ideal values

- Step 2, where parameters $S f R_{1}$ and $S f R_{2}$ are optimized. At this step, ideal values of $g_{k q 1,2}$ are used, and $R_{\text {gain }}$ and OBG are set to the values obtained at step 1

- Step 3, where the parameters $g_{k q 1,2}$ are optimized and the rest of parameters are fixed to the values determined at the previous steps.

Fig. 3 illustrates the above step-by-step optimization procedure by depicting some parametric analysis carried out at steps 1 and 2. At each optimization step, a SIMSIDES model of the modulator shown in Fig. 2 is simulated and initial ranges considered for the design variables are heuristically set based on the designers experience. The design parameters are selected according to the trade-off obtained between SNDR, $P$, and stability. Table I summarizes the results of this optimization procedure by showing the values of selected design parameters for each standard and operation mode targeted by the modulator. One of the key strategies to minimize $P$ consists of reducing the values of $k_{i}$ coefficients, which is compensated by increasing the gain of the active adder in order to obtain the necessary loop-filter gain. The values of main circuit elements, i.e. transconductors, capacitors and inductors can be computed from the coefficient values shown in Table I by using the expressions described above, and are used to start the design of the modulator subcircuits.

\section{Circuit-Level Reconfiguration Techniques}

The main subcircuits of the BP CT- $\Sigma \Delta \mathrm{M}$ in Fig. 2 are the $\mathrm{Gm}-\mathrm{LC}$ resonators, the active adder and the 4-bit quantizer. The latter is implemented by using a conventional 4-bit flash ADC made up of a resistor ladder and regenerative-latch based comparators and a feedback current-steering DAC that uses cascode current cells to improve the output resistance. Special care is put in the reconfiguration and design strategies applied to the resonators and the active adder, since they are key factors to optimize the performance in terms of reconfigurability, power consumption and robustness to circuit variations. 


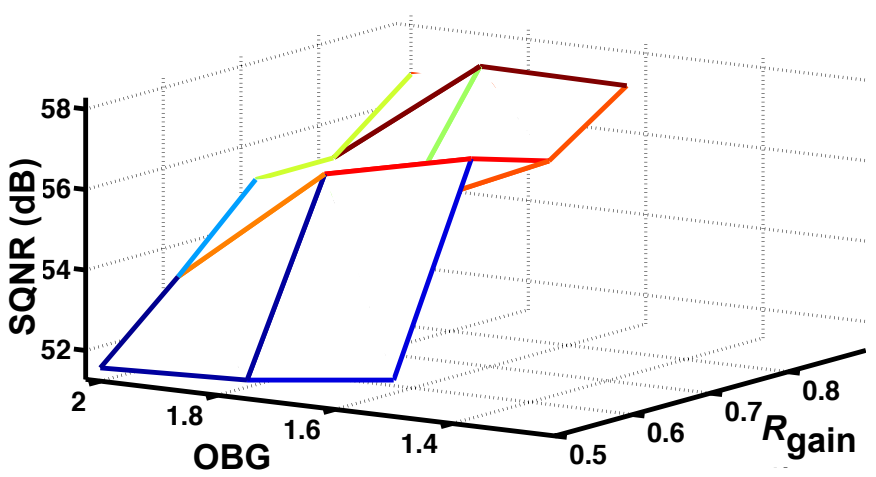

(a)

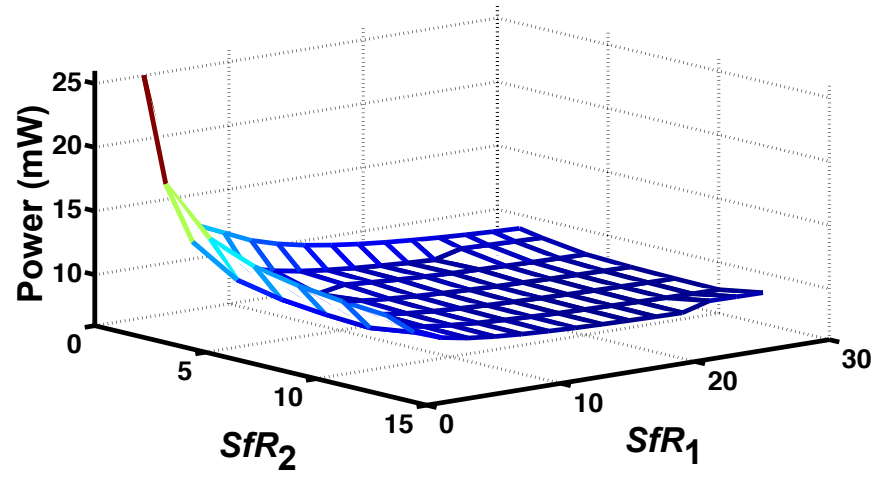

(b)

Fig. 3. Optimization procedure. (a) SQDR vs. OBG, $R_{\text {gain }}$ (b) $P$ vs. $S f R_{1,2}$.

Fig. 4(a) shows a conceptual schematic of the Gm-LC resonators. The circuit is made up of an LC tank and reconfigurable transconductors - depicted in Fig. 4(b). The forwardpath transconductors correspond to $g_{m 1,2}$ in Fig. 2 and are used to process the resonator input signals, while feedback transconductors implement $g_{k q 1,2}$ coefficients in Fig. 2. In both cases, the values of required transconductances are obtained by connecting switchable unitary inverter-based transconductors, so that depending on the operation mode in which the modulator is working, these unitary elements are either switched on or off (and powered down) in order to obtain the required ADC performance with the minimum power consumption.

A similar strategy is followed for the capacitors used in the LC tank, where a number of unitary MiM capacitors are connected through CMOS switches, which are controlled by digital logic in order to program the required notch frequency. An MOS varactor is also used to fine-tune the required resonance frequency needed in each case.

The analog active adder is one of the most critical building blocks of the BP CT- $\Sigma \Delta \mathrm{M}$ since it has to sum and amplify signals within the $\mathrm{GHz}$ range. This circuit has been designed to have a nominal gain of 64 in order to reduce the rest of loop-filter coefficients, with the subsequent benefits in terms of input/output swing, power dissipation and simplicity in the

TABLE I

HIGH-LEVEL SYNTHESIS OF THE BP CT- $\Sigma \Delta \mathrm{M}$

Loop-Filter Coefficients

\begin{tabular}{|c|c|c|c|c|c|c|}
\hline & $c_{0}$ & $c_{0 d}$ & $c_{1}$ & $c_{1 d}$ & $c_{2}$ & $c_{2 d}$ \\
\hline CDMA-450 & -0.62 & 1.22 & -2.92 & 2.76 & 1.72 & -1.28 \\
\hline LTE-700 & -0.73 & 1.33 & -3.32 & 2.10 & 1.48 & -2.07 \\
\hline GSM-900 & -0.46 & 0.80 & 0 & -3.32 & -3.12 & -1.28 \\
\hline \multicolumn{7}{|c|}{ System-Level Design Parameters } \\
\hline & OBG & $S f R_{1}$ & $S f R_{2}$ & $R_{\text {gain }}$ & $g_{k q 1}(\mathrm{mS})$ & $g_{k q 2}(\mathrm{mS})$ \\
\hline CDMA-450 & 2.5 & 16 & 4 & 1 & 5.8 & 5.8 \\
\hline LTE-700 & 2.5 & 16 & 4 & 1 & 2.0 & 2.0 \\
\hline GSM-900 & 2.5 & 16 & 4 & 0.8 & 1.6 & 1.6 \\
\hline \multicolumn{7}{|c|}{ Signal and Circuit Parameters } \\
\hline & $\overline{f_{n}(\mathrm{GHz})}$ & $f_{s}(\mathrm{GHz})$ & $L(\mathrm{nH})$ & $C(\mathrm{pF})$ & $R(\mathrm{k} \Omega)$ & $Q$ \\
\hline CDMA-450 & 0.45 & 1.25 & 6.7 & 18.6 & 1000 & 8.8 \\
\hline LTE-700 & 0.75 & 2 & 6.9 & 6.5 & 50 & 12.4 \\
\hline GSM-900 & 0.95 & 2 & 7.1 & 4.0 & 50 & 14.1 \\
\hline
\end{tabular}

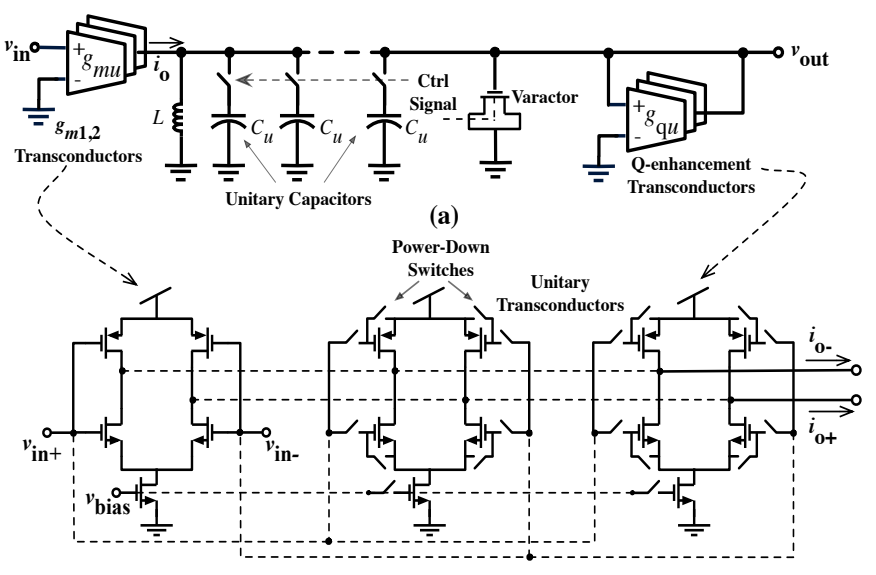

(b)

Fig. 4. Conceptual schematic of: (a) resonators and (b) transconductors.

design of the transconductors described above. The price to pay is that a more complex circuit is needed to implement the adder. In order to achieve the required gain, a fourth-stage amplifier has been considered. Each stage is made up of a transconductor based on a similar topology to that shown in Fig. 4(b) and a transImpedance amplifier. This adder circuit constitutes one of the design bottlenecks of the presented modulator, by consuming a significant part of its overall power consumption.

\section{Layout and Simulation Results}

The modulator has been implemented in a $65-\mathrm{nm}$ CMOS technology. Fig. 5 shows the complete layout of the chip, highlighting their main parts. The modulator core occupies an active area of $1.55 \times 1.55 \mathrm{~mm}^{2}$, and the layout floorplan considers separate analog, mixed and digital supplies, as well as guard-rings surrounding each section of the circuit. The area of the analog section is mostly devoted to the transconductors, inductors, capacitors, varactors, active adder, feedback DAC current sources and master-bias current generator. Comparator latches and switches are included in the mixed-signal section, while the digital part encloses the clock-phase generator and the required digital control/reconfiguration logic, that includes 


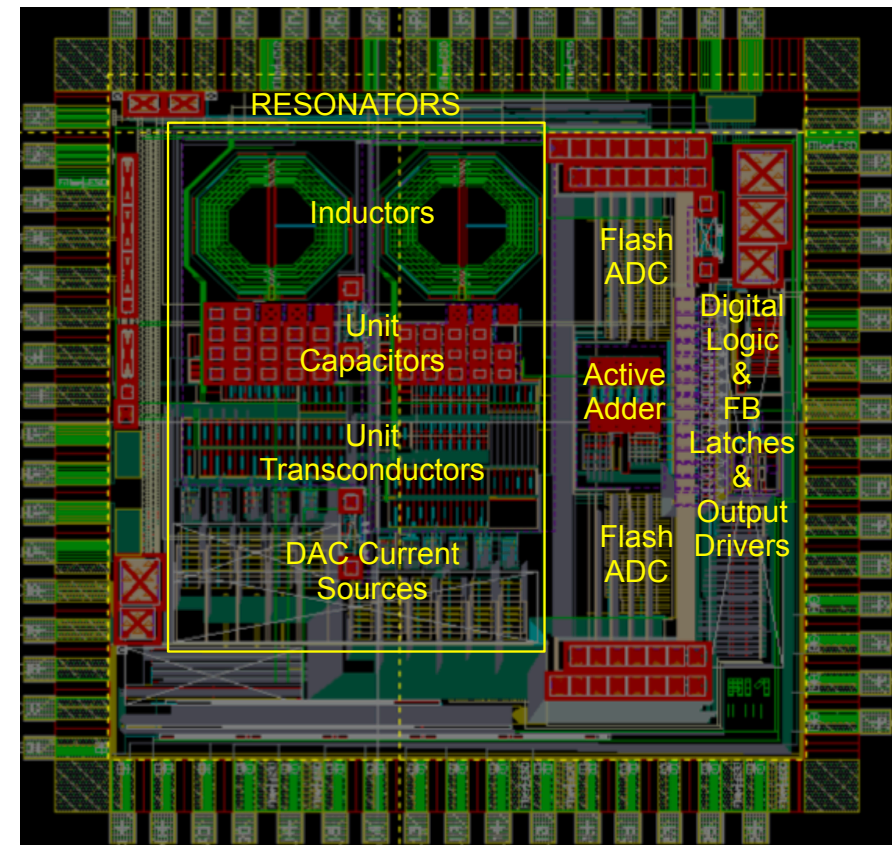

Fig. 5. Chip layout highlighting their main building blocks.

a ROM and a 4-bit parallel-to-serial register to transform the 4-bit $1.25 / 2 \mathrm{GHz}$ output bitstream into a 16-bit digital output clocked at $500 \mathrm{MHz}$ to simplify the test. Calibration techniques have been also included in the chip in order to separately test critical building blocks of the modulator, i.e. resonators, adder and quantizer. To this end, on-chip test buffers and switches can be configured to check the resonators outputs as well as their corresponding input test signals.

As an illustration, Fig. 6 shows the output spectrum of the modulator corresponding to the CDMA operation mode. Finally, Table II summarizes the results of the modulator and compares its simulated performance with the state of the art in BP- $\Sigma \Delta \mathrm{Ms}$ with programmable notch. Note that the presented design features the lowest power consumption, while keeping a competitive performance in terms of $B_{w}$, DR and Figureof-Merit $^{2}$ (FOM).

\section{CONCLUSIONS}

The design of a Gm-LC fourth-order BP CT- $\Sigma \Delta \mathrm{M}$ in $65-\mathrm{nm}$ CMOS has been presented. A key contribution of this design is based on a systematic synthesis methodology which optimizes the loop-filter coefficients at system level, and the transconductor implementation at circuit level, in order to efficiently digitize RF signals with $40-\mathrm{MHz}$ bandwidth placed at carrier frequencies within the 450 -to- $950 \mathrm{MHz}$ band, while clocked at $1.25 / 2 \mathrm{GHz}$. These results show the potential effectiveness of the presented techniques for practical implementation of SDRs and next-generation mobile systems.

${ }^{2} \mathrm{FOM}$ metrics in Table II are defined as: FOMw $\equiv \frac{P(\mathrm{~W})}{2 \cdot B_{w}(\mathrm{~Hz}) \cdot 2^{\mathrm{ENOB}(\mathrm{bit})}}$ and FOMs $\equiv \mathrm{DR}(\mathrm{dB})+10 \cdot \log _{10}\left[B_{w}(\mathrm{~Hz}) / P(\mathrm{~W})\right]$, where ENOB stands for Effective Number Of Bits.

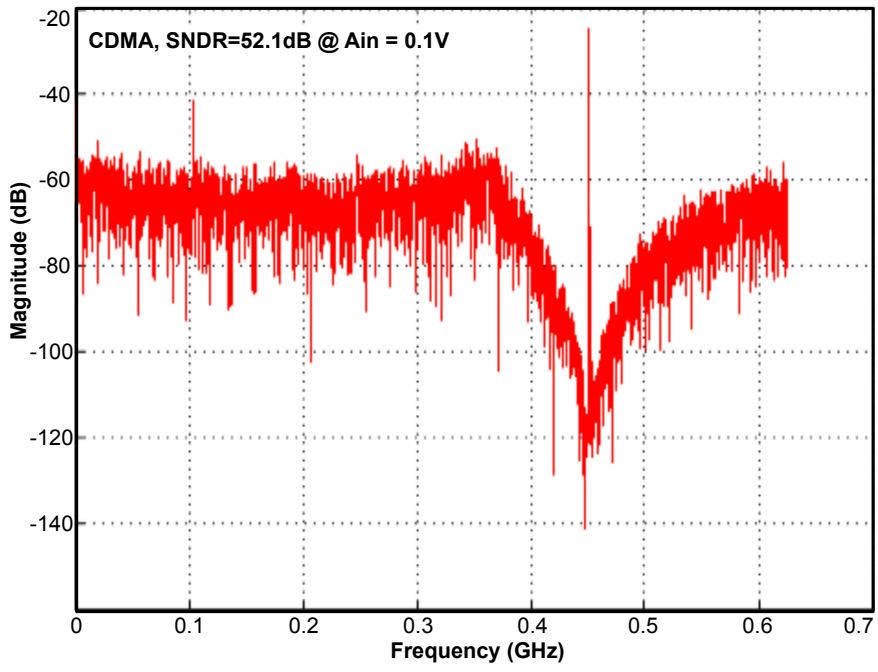

Fig. 6. Output spectrum of the modulator for CDMA standard.

\section{REFERENCES}

[1] K. Koli et al., "A 900-MHz Direct Delta-Sigma Receiver in 65-nm CMOS," IEEE J. of Solid-State Circuits, pp. 2807-2818, Dec. 2010.

[2] M. Bolatkale et al., "A $4 \mathrm{GHz}$ Continuous-Time $\Delta \Sigma$ ADC With $70 \mathrm{~dB}$ DR and -74 dBFS THD in $125 \mathrm{MHz}$ BW," IEEE J. of Solid-State Circuits, vol. 46, pp. 2857-2868, December 2011.

[3] P. Shettigar and S. Pavan, "Design Techniques for Wideband Single-Bit Continuous-Time $\Delta \Sigma$ Modulators With FIR Feedback DACs," IEEE J. of Solid-State Circuits, vol. 47, pp. 2865-2879, December 2012.

[4] H. Shibata et al., "A DC-to- $1 \mathrm{GHz}$ Tunable RF $\Delta \Sigma$ ADC Achieving $\mathrm{DR}=74 \mathrm{~dB}$ and $\mathrm{BW}=150 \mathrm{MHz}$ at $f_{0}=450 \mathrm{MHz}$ Using $550 \mathrm{~mW}$," IEEE ISSCC Digest of Technical Papers, pp. 150-151, February 2012.

[5] E. Martens et al., "RF-to-Baseband Digitization in 40nm CMOS With RF Bandpass $\Delta \Sigma$ Modulator and Polyphase Decimation Filter," IEEE J. of Solid-State Circuits, vol. 47, pp. 990-1002, April 2012.

[6] S. Gupta et al., "A 0.8-2GHz Fully-Integrated QPLL-Timed Direct-RFSampling Bandpass $\Sigma \Delta$ ADC in $0.13 \mu \mathrm{m}$ CMOS," IEEE J. of Solid-State Circuits, vol. 47, pp. 1141-1153, May 2012.

[7] M. Englund et al., "A Programmable $0.7-2.7 \mathrm{GHz}$ Direct $\Delta \Sigma$ receiver in 40nm cmos," IEEE J. of Solid-State Circ., pp. 644-655, March 2015.

[8] A. Ashry and H. Aboushady, "A 4th Order 3.6 GS/s RF $\Sigma \Delta$ ADC With a FoM of 1pJ/bit," IEEE Trans. on Circuits and Systems - I: Regular Papers, vol. 60, pp. 2606-2617, October 2013.

[9] H. Chae et al., "A $12 \mathrm{~mW}$ Low Power Continuous-Time Bandpass $\Delta \Sigma$ Modulator With $58 \mathrm{~dB}$ SNDR and $24 \mathrm{MHz}$ Bandwidth at $200 \mathrm{MHz}$ IF," IEEE J. of Solid-State Circuits, vol. 49, pp. 405-415, February 2014.

[10] C. Wu et al., "A Wideband $400 \mathrm{MHz}-$ to-4 GHz Direct RF-to-Digital Multimode $\Delta \Sigma$ Receiver," IEEE J. of Solid-State Circuits, vol. 49, pp. 1639-1652, July 2014.

[11] G. Molina et al., "LC-Based Bandpass Continuous-Time Sigma-Delta Modulators with Widely Tunable Notch Frequency," IEEE Trans. on Circuits and Systems - I: Regular Papers, pp. 1442-1455, May 2014.

[12] J. Ruiz-Amaya et al., "High-Level Synthesis of Switched-Capacitor, Switched-Current and Continuous-Time $\Sigma \Delta$ Modulators Using SIMULINK-based Time-Domain Behavioral Models," IEEE Trans. on Circuits and Systems - I: Regular Papers, pp. 1795-1810, Sep. 2005.

TABLE II

Simulated Performance Summary

\begin{tabular}{|c|c|c|c|c|c|c|}
\hline & $f_{n}, f_{s}(\mathrm{GHz})$ & $B_{w}(\mathrm{MHz})$ & $\mathrm{DR}(\mathrm{dB})$ & $P(\mathrm{~mW})$ & $\mathrm{FOMw}(\mathrm{pJ} / \mathrm{c})$ & $\mathrm{FOMs}(\mathrm{dB})$ \\
\hline CDMA & $0.45,1.25$ & & 50 & 22.7 & 1.11 & 142 \\
LTE & $0.75,2.0$ & 40 & 57 & 22.8 & 0.48 & 149 \\
GSM & $0.95,2.0$ & & 56 & 16.6 & 0.41 & 150 \\
\hline
\end{tabular}
\begin{tabular}{|c|c|c|c|c|c|c|}
\hline State-of-the-Art BP- $\Sigma \Delta$ Ms with Programmable Notch \\
\hline$[6]$ & $(0-1),(2-4)$ & 150 & 69 & 550 & 0.8 & 153 \\
\hline$[7]$ & $(0.8-2),(2.6-3.4)$ & 1 & 46 & 30 & 92.0 & 121 \\
\hline$[10]$ & $(0.7-2.7),(1.25)$ & 15 & 43 & 90 & 26.9 & 103 \\
\hline
\end{tabular}

